\title{
VW HYDRI, A U GEMINORUM-TYPE STAR WITH PERIODIC VARIATIONS DURING OUTBURSTS
}

\author{
N. VOGT \\ European Southern Observatory, La Silla, Chile
}

\begin{abstract}
Extensive photoelectric observations of VW Hyi have been carried out during minimum light and eruptions. In the minimum stage VW Hyi shows a periodically repeating hump (similar to that of $\mathrm{U}$ Gem) from which an orbital period of 0.07427111 has been derived. No eclipses are present. The two types of eruptions known previously for VW Hyi correspond to different physical processes: the short eruptions (lasting $\sim 4^{d}$ ) resemble again those of $U$ Gem; the minimum hump disappears during bright phases. During the long eruptions (lasting $\sim 17^{\mathrm{d}}$ ) a light curve with pronounced peaks has been observed with periodicities of $\sim 0$ d.0768. Significant colour variations with this period are also present. During one long maximum, the repeating feature separated into two peaks. A first attempt has been made to interpret qualitatively the observed facts by an interaction between gas streams from the inner Lagrangian point and ejected matter from the white dwarf component.
\end{abstract}

The detailed results were published in Astronomy and Astrophysics 36 (1974), 369. 